\title{
ARTICULACIONES PÚBLICO-PRIVADA PARA LA OFERTA EDUCATIVA: ENCANTAMIENTOS, SOSPECHAS, TENSIONES
}

\author{
Alfredo Astorga*
}

\begin{abstract}
RESUMEN: El tema de articulaciones público-privadas se ha extendido, especialmente en educación, con más discurso que prácticas. Entre los actores los desacuerdos superan todavía a las convergencias. La visión de lo público, como lo colectivo y no solo estatal, facilita las articulaciones al colocar a todos los actores como responsables, con roles particulares, insustituibles. En educación, bien público por excelencia, se abren caminos para una nueva cultura de diálogo entre actores del Estado, sector empresarial y sociedad civil. Es preciso disolver encantamientos y sospechas para afrontar las articulaciones, transparentando su dimensión política. La defensa de los derechos constituye el corazón de las articulaciones y la exigibilidad - que implica participación integral y permanente - su línea maestra. Este enfoque confiere a las articulaciones un carácter estratégico y posibilita encarnar los derechos en las grandes y cotidianas conquistas por la calidad-equidad en la educación.
\end{abstract}

Palabras clave: Articulación estratégica. Responsabilidad social empresarial. Políticas públicas. Derecho a la educación. Exigibilidad.

\section{PUBLIC-PRIVATE PARTNERSHIPS FOR PROVISION OF EDUCATION: ENCHANTMENTS, SUSPICIONS, TENSIONS}

ABSTRACT: The theme of public-private partnerships, especially in education, has been disseminated much more through speeches than practice. Among players, disagreements far outnumber convergences. Seeing public as collective, not only state-owned,

Oficina Regional de Educação para América Latina e Caribe (oreAlc/unesco). E-mail: alfastorga@email.com

Educ. Soc., Campinas, vol. 30, n. 108, p. 699-715, out. 2009 
facilitates partnerships, since all players thus become responsible and are given particular, irreplaceable roles. In education, the common good par excellence, paths have been developed that allow for a new dialog culture among the State, entrepreneurs and the civil society. Enchantments and suspicions must be allayed to deal with these partnerships and reveal their political dimension. Defending rights is at the heart of such partnerships and enforceability - which implies total and permanent participation - is their master line. Such focus endows these partnerships with a strategic feature and allows to insert rights among the important, daily conquests towards quality-equity in education.

Key words: Strategic partnership. Corporate social responsibility. Public policies. Right to education. Enforceability.

\section{Marcando la cancha}

$E$ 1 tema de articulaciones ${ }^{1}$ entre la esfera pública y privada, por su naturaleza polémica, su grado de desarrollo y variantes, está aun plagado de supuestos, riesgos y tendencias no consolidadas. Por ello resulta clave delimitar una comprensión de partida - aunque no sea exhaustiva ni definitiva - que sirva de referente. En Educación es doblemente válida dada la profusión de experiencias y lecturas, y dado el carácter estratégico del sector. ${ }^{2}$

Respecto de lo público, que refiere al poder instituido histórica, oficial y legalmente y se expresa a través de los poderes e instituciones del Estado, destacamos algunas tensiones: el alcance de lo público, sobre todo a nivel de subunidades territoriales que conectan con instancias sociales; la representación y mecanismos de presencia ciudadana en ciertos parlamentos y partidos políticos; la natural existencia de diversos intereses. Vale mencionar que en los últimos años asistimos a profundos cambios en la comprensión (y acción) sobre lo público, que van de la mano de enormes desilusiones de la democracia formal.

En la esfera de lo no-público, diferenciamos ante todo sector privado empresarial y sociedad civil. Su frontera esencial: el lucro en el primer caso, el bienestar social en el segundo.

Esta categorización muestra también tensiones. En el sector privado empresarial, el alcance es amplio y complejo. No es lo mismo 
una transnacional, una empresa mixta, una microempresa, un emprendimiento familiar, un autoempleo o una empresa comunitaria, aunque todas busquen optimizar las ganancias. Las últimas sobre todo - empresas comunitarias con múltiples rostros en AL - hacen tambalear algunas definiciones tradicionales.

En la sociedad civil - que incluyen al menos colectivos territoriales, organizaciones sociales, movimientos ciudadanos, ${ }^{3}$ ONGs, redes, corrientes de la iglesia, instancias de derechos - resaltamos tres nudos: la representación; la pertenencia simultánea a varios espacios con institucionalidad diversa; la existencia de sectores no organizados o muy aislados y anónimos.

Las delimitaciones planteadas son aplicables al campo de la $E d u$ cación. Añadimos que a nivel de lo público hay un predominio del poder ejecutivo y en él de los Ministerios de Educación y Hacienda. ${ }^{4}$ El involucramiento de otros ministerios sociales, de planificación y del gobierno como conjunto suele ser secundario, al igual que la de los parlamentos, aunque en estos se visualiza un interés inusitado por el debate y el seguimiento. Los gobiernos locales ganan protagonismo; planes locales integrales de educación con aval ciudadano surgen en muchos países - andinos y centroamericanos entre ellos -, especialmente en el campo y pequeñas urbes.

La participación del sector privado empresarial, en general, continúa siendo dispersa, puntual, acotada al quehacer de las empresas y con enfoques asociados a la filantropía o cargados al posicionamiento de imagen. Unas pocas empresas han ingresado en políticas de mediano alcance en becas, educación infantil, lectura, formación para el trabajo. ${ }^{5}$

En la sociedad civil la presencia de organizaciones de carácter amplio como frentes, foros ciudadanos y observatorios han crecido en presencia y capacidad de crítica y propuesta. Es importante señalar el carácter aglutinador de estos nuevos movimientos que integran individuos y organizaciones. Es notoria la presencia de ONG en campos sensibles: alfabetización y educación para jóvenes y adultos, educación alternativa, formación para el trabajo, ejes transversales sobre derechos, violencia, interculturalidad, etc. La sociedad civil es ya un actor imprescindible, aunque su participación en decisiones de política tenga límites. 
Articulaciones público-privada para la oferta educativa...

Nos enfocaremos en las intervenciones del sector privado empresarial en la educación.

\section{Discurso en busca de práctica}

El tema de las alianzas público-privadas se ha expandido como mancha de aceite en las últimas décadas. Casi no hay discurso público que no mencione sus bondades y apunte a su consecución. ${ }^{6}$ Junto a un valioso signo por vigorizar las democracias gastadas, subyacen tendencias que muestran que el discurso está sobredesarrollado en relación a las prácticas; las palabras han caminado más rápido que las experiencias. Y ello genera encantamientos, distorsiones... La historia sigue marcada más por un vaivén de encuentros y desencuentros entre actores que por una convergencia perdurable.

Esto es aplicable en muchas esferas y particularmente en educación. Más significativo aun considerando que no es ella la que más estimula la participación de los privados. La salud, la cultura, el entretenimiento o el deporte generan mayor visibilidad e impactos más rápidos. De todas maneras, toda generalización sería un error pues coexisten en las relaciones de la empresa con el mundo público diversas tendencias, unas en que predominan la separación y autonomía y otras en que prevalecen la convergencia, los complementos y los acuerdos explícitos.

Entre las primeras se puede mencionar la oferta privada de educación, la cual - con excepción de Cuba - ha mostrado crecimiento constante, de la mano de los modelos privatizadores (en Chile representa cerca del $10 \%$ de la oferta). Si bien cuenta con normativas y estímulos favorables, suele desarrollar su propuesta con alto grado de autonomía y bajo las lógicas del mercado. La calidad del servicio se asocia al costo de la oferta, prohibitivo para las mayorías. La aproximación hacia modelos de países desarrollados y estándares internacionales es la constante (parámetros de la OCDE, por ejemplo). Esta oferta - territorios libres de Estado - difícilmente puede calificarse como expresión de una alianza público-privada, aunque se sustenta en acuerdos tácitos.

En esta línea se ubican también los aportes que realiza la empresa privada en forma puntual y dispersa a las instituciones educativas: 
materiales, infraestructura, equipamiento, actividades culturales y recreativas, estímulos y premios, etc. Estos aportes no obedecen a planes sistemáticos de colaboración, sino a gestión de autoridades o iniciativa de empresas en entornos de su interés (para ganar imagen o ampliar consumidores, por ejemplo).

En la ruta de convergencias resaltamos, en primer lugar, las ofertas combinadas público-privadas llamadas escuelas subvencionadas o de financiamiento compartido. Empresarios privados (a veces cadenas) cuya inversión se enriquece con aportes públicos en pago de profesores o subsidios por alumno. El servicio tiene costo para las familias, aunque menor que el de escuelas privadas. En varios países esta modalidad representa un gran negocio con escaso riesgo. En otros, como en los andinos, esta variante está administrada por instancias de la iglesia social o fundaciones sin fines de lucro y se dirige hacia sectores con bajos recursos (el caso de Fe y Alegría es uno de los más emblemáticos y mejor evaluados). El alineamiento con políticas públicas suele ser mayor. Lastimosamente esta articulación no es explotada como tal; los acuerdos se limitan muchas veces a los aportes financieros. El modelo se parece más a un auxilio al Estado en sectores prioritarios, con el peligro de sustituir sus roles indelegables.

En segundo lugar, identificamos la vigorosa presencia de la llamada responsabilidad social empresarial (RSE) ${ }^{7}$ o corporativa, que está en pleno proceso de consolidación y diferenciación interna. La adhesión a la RSE se instala como una necesidad de eficacia y sostenibilidad del negocio, pero sin perder la lógica empresarial y de competencia, o en función de ellas se desarrollan nuevos roles ligados a aportes sociales y medio ambientales, unas veces ligados estrechamente a su quehacer (por ejemplo, empresas de telecomunicaciones que aportan en introducción de computadores) y otras no (por ejemplo, empresas telefónicas que impulsan campañas para reducir el trabajo infantil). El tema amerita ya investigaciones y estudios particulares porque igual están presentes muchos mitos, críticas y visiones triunfalistas. Ciertos niveles de suspicacia y desconfianza seguirán presentes mientras la práctica empiece a demostrar vigorosamente tendencias inequívocas. ${ }^{8}$

Por lo mencionado, reafirmamos que en el campo de las alianzas público-privadas florece la diversidad y no es la articulación la señal dominante en el campo de la educación. Las líneas más progresistas y 
minoritarias de la RSE - como filosofía y como práctica - están generando y/o aceptando articulaciones programáticas de plazos más largos e impactos duraderos. Algunos temas que actualmente se trabajan con este sentido son la educación técnica y vocacional, la masificación del idioma inglés, la generalización de TIC, la educación infantil.

Es interesante mencionar que muchas de las empresas adherentes a la RSE participan cada vez más en los foros amplios que instituciones regionales o mundiales - como UNESCO, OEI, OEA - fomentan periódicamente. ${ }^{9}$ Los actores empresariales privados fortalecen cada vez más su rol como interlocutores, con derecho y deber de participar en una esfera social como la educativa, que antes parecía propiedad exclusiva de autoridades y maestros. Y ya no es solo el campo de la educación profesional donde su voz parecía no solo razonable sino necesaria, es también sobre áreas tan diversas como la educación infantil y la educación no formal.

\section{Articulaciones y derecho a la educación}

La visión anterior merece complementarse con una pequeña reflexión sobre dos enfoques que tiene aun peso importante en las alianzas desde el sector empresarial privado: uno de carácter mercantilista y otro filantrópico.

En el enfoque que llamamos mercantilista, el corazón y sentido de los acuerdos están dados exclusivamente por la posibilidad de mejorar el posicionamiento público, la imagen de marca y la reputación, que a la larga se convierten en ventajas comparativas para la competencia. Esta corriente está presente aun en propuestas que se cobijan bajo la RSE y tienen diversas expresiones en el campo de la educación. La mayoría de ellas se caracterizan por hablar de "donaciones" que se concentran sobre todo en "cosas", productos tangibles y prestan escasa atención a procesos y mucho peor de mediano o largo plazo. En esta línea podríamos ubicar los múltiples mini proyectos de entrega de materiales, computadores, equipos, uniformes, entre otros, que sin ser incorrectos en sí mismos, no se insertan en procesos de mejoramiento. No es casual además que estas donaciones se entreguen por una solo vez y que algunas empresas alteren cada año el destinatario, niños pobres, niños con necesidades especiales, etc. 
En el fondo, estas iniciativas convierten el paradigma de la RSE solo en oportunidad de negocio. El destinatario no tiene rostro y no tiene sentido de sujeto portador de derechos. El despliegue publicitario suele ser una de las actividades más relevantes y en ocasiones costosas. El efecto buscado de adhesión, reconocimiento y fidelidad muchas veces se logra. En términos de transformación educativa, esta forma de entender las alianzas aporta muy poco.

El enfoque filantrópico (explícito o encubierto) baña muchas experiencias privadas que participan en alianzas público-privadas en la educación. Efectivamente, la mayoría de discursos (desde el Estado y la sociedad) identifica estos aportes como "desprendimientos generosos" de las empresas a favor de los necesitados, y que complementan a los Estados que muestran limitaciones. La alusión a principios morales o religiosos suelen estar presente, añadiendo un tinte de "caridad y compasión" a estos apoyos.

Es preciso, en primer lugar, desmitificar estas argumentaciones. Las empresas han ingresado al campo de las políticas sociales no por razones de piedad o caridad. Lo han hecho como una manera distinta y mejor de posicionar en los mercados las marcas, las imágenes corporativas. Es una modalidad que en algunos casos se plantea ampliar mercados o reducir imposiciones. El Fondo de Educación del BSR señala entre los beneficios: mejoramiento del desempeño financiero, reducción de costos operativos, mejora de la imagen de marca y la reputación. Todo esto resulta legítimo y natural a la lógica empresarial y no precisa por tanto encubrirse bajo el manto de donaciones, sacrificios, reducción de utilidades por solidaridad, etc.

Lo cuestionable reside en la noción de la "caridad" que se utiliza, la idea del regalo a los necesitados. Esta visión instala la idea de la compensación o la "salvación" por ayuda de otros y no por derecho y esfuerzo propio, dejando tras de sí una estela de estigmatizaciones y una sensación de desprotección y exclusión de las riquezas de una sociedad.

La función esencial del Estado como garante del derecho a la educación queda tambaleando, pero también queda exculpado de alguna manera, con el argumento de sus limitaciones. Los aportes así concebidos disminuirán los esfuerzos que debe hacer el Estado para procurar recursos y estrategias que permitan cumplir su rol; 
Articulaciones público-privada para la oferta educativa...

los aportes externos en educación bajarán la presión al Estado en su cambio de prioridades, en el rigor del cumplimiento de compromisos internacionales con metas educativas.

Los principios y compromisos por la gratuidad de la educación, de la universalización de la enseñanza con calidad quedan reducidos a la buena voluntad. Su carácter imperativo y su dimensión ética y política tienden a disolverse.

En un enfoque de derechos, el sentido de las articulaciones público-privadas adquiere connotaciones muy diversas y no restan importancia a los principios y compromisos. Resaltan el rol insustituible de garante del Estado, entienden las colaboraciones como deberes y compromisos, conciben a los destinatarios como seres humanos sujetos de derechos. No dejan espacio para los estigmas ni el voluntarismo.

Estas prácticas filantrópicas no dejan ver con toda su crudeza los problemas de desigualdad y exclusión, que tienen en la educación un área clara de expresión. Finalmente auspician la entrega voluntaria de sobrantes sin cuestionar el por qué un sector puede darse el lujo de tener sobrantes, mientras las mayorías no alcanzan a cubrir sus mínimas necesidades. Algo funciona mal en las profundidades de la sociedad, algo que tiene que ver con el reparto de la riqueza, que no es lo mismo que el reparto de los sobrantes generados en la mala distribución de la misma. La calidad diferente de la educación de acuerdo al costo del servicio no terminará con las donaciones y paliativos, sino cuando estructuralmente no se permita ofertas tan disímiles.

Si es cierto que la educación es un bien público (de todos) y estratégico (imprescindible para el desarrollo), resulta inaceptable que exista educación de primera y de segunda categoría. Un contrasentido que no podrá compensarse con aportes sobrantes del sector empresarial privado, sino con un cambio radical de políticas por parte de los Estados. El ejercicio del derecho a la educación y su consecuencia con él subyacen en el fondo de estas reflexiones.

\section{Descomprimiendo lo público}

Uno de los saltos más interesantes en términos conceptuales, políticos y éticos alude a las nuevas comprensiones que se construyen sobre el sentido de lo público. La visión predominante asociaba 
excluyentemente lo público con lo estatal. La "esfera de todos" estaba comprimida, entregada mediante un voto periódico, y casi siempre sin posibilidad para el arrepentimiento, al Estado y sus gerentes. La educación, no ha sido la excepción; la educación como tarea de toda una sociedad no ha sido la perspectiva dominante; la delegación en el Estado ha sido el sello predominante.

Pero el movimiento ha continuado. Lo público ha empezado a identificarse cada vez más claramente como "lo de todos", lo colectivo, lo que nos compete irremediablemente y cuyo manejo entraña nuevos deberes y desafíos para la participación. La delegación como cheque en blanco, por la vía exclusiva del voto, está tocando fondo y empieza a renovarse con el principio de la responsabilidad ciudadana. Una participación que trae en su esencia el sentido de derecho y al mismo tiempo el sentido de compromiso y deber. Varias de las últimas declaraciones en educación insisten en el lema de la "educación compromiso de todos", para enfatizar justamente la corresponsabilidad, la necesidad de avanzar desde las políticas educativas de ministerio hacia las políticas educativas de gobierno, de Estado y de sociedad.

La intervención en lo público se amplía con estas nuevas nociones. Supera con creces el voto periódico para volverse acción, presión, tensión permanente. Se atreve a incorporar todas las voces al campo de las políticas públicas, antes reservadas a las élites gobernantes de cualquier signo. La participación en el destino colectivo adquiere rango de derecho humano inalienable, complemento y facilitador del conjunto de derechos de los seres humanos, individual y colectivamente considerados. La democracia intenta volverse cotidiana, tensarse hasta sus límites, jugar en todos los espacios, tornarse símbolo y vida.

En el campo educativo la participación también avanza hacia todos los ámbitos, desde la institución escolar hasta las políticas educativas y los convocados se amplían sin límites; entonces cobra sentido la palabra no solo de maestros, estudiantes y familias, sino también de organizaciones sociales, ONGs, mundo académico, mundo de la empresa privada. Y a nivel del Estado, los ministerios sociales, los parlamentos, los gobiernos locales.

También aquí las delimitaciones son imprescindibles y sobre todo respecto a roles. Cuando tratamos las articulaciones estratégicas, hablamos más allá de la suma o agregado de fuerzas y funciones 
Articulaciones público-privada para la oferta educativa...

similares. Hablamos de convergencia e integración en un conjunto, de actores con roles diversos, lo que multiplica la potencialidad tanto como la complejidad.

La nueva visión trae aparejada la necesidad de reinventar el Estado para que tenga soporte como único garante de los derechos de todos. Y esto como un rol insustituible, una esencia de la función. Ninguna otra función o actor tiene las condiciones ni mandatos para cumplir tan delicada y extendida labor. El tema y las experiencias nos lleva también a repensar el tema del tamaño y fuerza del Estado para cumplir sus roles. Creemos que es hora de trascender el debate entre Estado minúsculo, solo facilitador del mercado vs. Estado omnipresente, obeso e interventor en todos los espacios.

La discusión, felizmente, está moviendo su centro de gravedad... ni Estado obeso ni Estado ausente. Optamos por la versión de "Estado suficiente". Y suficiente para cumplir su esencia: la garantía de derechos para todos. El tamaño y la fuerza están en función del rol que se define en cada contexto. Por eso la homogenización de modelos en sociedades de tan diverso grado de desarrollo y combinación cultural resulta un contrasentido. La persistencia de la pobreza y sus peores caras de exclusión e inequidad refuerza un deber ético que sobrepasa al sector público estatal. Y con ello no solo estimula acuerdos sino que también incentiva el desarrollo de concepciones nuevas de democracia, de participación, de ejercicio ciudadano.

En el campo de la educación la idea del Estado suficiente está marcada por su responsabilidad para garantizar el derecho a la educación, entendido como derecho al aprendizaje de calidad sin exclusión de ninguna naturaleza y extensible a lo largo de toda la vida y en todas las circunstancias. (Nótese que la comprensión del derecho a la educación supera con creces al tradicional entendimiento de "estar en la escuela").

Sin duda el salto en las concepciones, visible apenas en algunas prácticas, tiene muchas implicaciones, imposibles de agotar en este texto. Al menos enfatizamos la necesidad de estudiar a profundidad, sistematizar prácticas e impulsar innovaciones desde esta nueva visión. Y también llamamos la atención por las modificaciones que implica en el campo de la cultura... una nueva forma de encarar el diálogo, las diferencias, los conflictos, las convergencias, los plazos, la lectura del contexto propio y global. 
Los riesgos sobre el tema son visibles. El reduccionismo en la visión de las alianzas, que minimiza su alcance o niega su carácter político para dejarlo en el área de la buena voluntad. El riesgo de retórica, cuando no pasa de las palabras, las campañas y las seudo consultas y no llega a encarnarse como democracia integral y permanente. El riesgo del exitismo basado en experiencias con poco sentido de sostenibilidad y alianza estratégica de sujetos.

\section{A recuperar la política}

La realidad evidencia con claridad creciente la posibilidad y necesidad de encuentros estratégicos entre sectores y actores directos en educación (autoridades, maestros, estudiantes, familias) y más allá (sector privado empresarial, sociedad civil). El discurso generado hace pocas décadas luce más sólido e integral, y parece encontrar mejores condiciones de aplicación y de encuentro con experiencias educativas relevantes. Un nuevo sentido de participación democrática empieza a colocarse en el imaginario de los actores que se involucran en la educación. Las decisiones educativas, pequeñas y grandes, empiezan a buscar oxígeno fuera del sector, empiezan a dejar su encierro para convertirse motivo de preocupación o beneplácito de una sociedad en conjunto. Las alianzas y articulaciones público-privadas finalmente tienen tras de sí este sentido de nueva participación, de ejercicio innovador de la democracia.

Estamos en un momento apropiado para develar los encantamientos de las alianzas en educación, más sustentados en la retórica y el coyunturalismo que tiende a simplificar procesos que son complejos, que tiende a vaciarlos de política y juego de intereses.

Es momento de desmantelar las sospechas y el escepticismo como signo pre existente de todo cambio y relación. Superar las sospechas no implica eliminación de críticas, pero sí apertura y transparencia para reconocer y trabajar con las diferencias, que en educación son innumerables y naturales. Si el encantamiento las minimiza, el escepticismo las sobredimensiona; y ambas obstaculizan finalmente la suma de esfuerzos y roles hacia objetivos comunes.

Es momento de admitir que el campo de juego de las alianzas en educación es y será la arena política. Es imperativo recuperar su sentido, su misión, su territorio complejo y cubierto de tensiones e 
Articulaciones público-privada para la oferta educativa...

intereses distintos, pero no por eso antinatural o inmovilizador. Es momento de reconocer que las alianzas de carácter estratégico en la educación se construyen permanentemente, con avances y retrocesos en medio de escenarios complejos y cambiantes. No son pactos de sangre que se producen una vez y para siempre. Son caminos turbulentos de encuentros y desencuentros, pero que han apostado por algunas luces de llegada.

El carácter natural de las diferencias no implica la repetición de ciclos similares sin aprendizaje. Aquí está uno de los desafíos claves, el sentido de aprendizaje desde la práctica, de acumulación de experiencia para que cada nuevo ciclo encuentre a los actores que se comprometen con la educación en diferente piso, en otra vuelta de espiral y no de círculo.

Esta sistematización requiere nuevos ingredientes: organizaciones que gestionan conocimientos y son capaces de aprender y seguir aprendiendo, instancias con apertura y flexibilidad para avanzar sólida y gradualmente, actitudes que, reconociendo diferencias, pueden avanzar en muchas aristas estratégicas. El blanco-negro, el todo-nada paralizan. Si es un camino hirviente, la respuesta no es negar el camino sino atreverse a seguir sobre las brasas.

Junto a la sistematización se hace necesario desplegar un esfuerzo sostenido de investigación de estas alianzas público-privadas. El conocimiento acumulado es relativamente pobre aunque las experiencias crecen y el discurso se inflama. Existen muy pocos datos integrales respecto de la inversión de los sectores privados en educación (más allá de las empresas educativas), los sujetos prioritarios a los que se destinan los esfuerzos, las áreas y temas que se privilegian, los resultados de corto y largo plazo de las intervenciones o aportes.

Las alianzas estratégicas más exitosas tienen un corazón y una esencia que las distingue: la conquista indeclinable por mayores espacios, profundidad y actores en ejercicio de sus derechos. Los derechos humanos, y el derecho a la educación de calidad para todos, debe volverse un eje articulador estratégico. Sin este piso y techo ético y político, las alianzas tienden a perderse en la coyuntura y el activismo. Esta alma de los acuerdos no subestima otras convergencias, pero sí las sitúa en una perspectiva distinta, posible de ser acogida por su 
alcance mundial, por el nivel de adscripción generalizada, por su esencia humanista, por su carácter de constituir el mejor resultado de la globalización.

En el sector de la Educación, el enfoque de derechos, revitalizado en los últimos tiempos y comprendido como el derecho a aprender con calidad a lo largo de la vida y sin discriminación de ninguna naturaleza, revela una agenda rica y sugerente para provocar acercamientos. ${ }^{10}$ Los procesos y resultados en educación en general quedan bajo las expectativas y las promesas y revelan descarnadamente la inequidad en acceso, en calidad, en resultados.

En esta mirada resulta esencial la concepción amplia de las articulaciones público-privadas. Es momento de abrir el espacio y no multiplicar esfuerzos bilaterales. Estos espacios están llamados para integrar sector público, sector privado empresarial y representaciones de la sociedad civil. En la convergencia de estos tres sectores descansa mucho de las posibilidades de éxito y sostenibilidad de las iniciativas.

En este mismo sentido, surge como la estrategia más brillante la llamada exigibilidad y justiciabilidad de los derechos. El simple reconocimiento de su valor es insuficiente y deja demasiado espacio para la retórica y el reduccionismo. Es esta lucha la que recupera y aterriza los derechos en su auténtica dimensión, los vuelve cotidianos, los vuelve de carne y hueso, los absorbe desde las esferas especulativas, academicistas o judicialistas en que pretenden que queden varios gobiernos y sectores. Es preciso rescatarlos de su encierro. Y estirar y tensar sus sentidos hasta las últimas consecuencias.... La exigibilidad como proceso social convergente, autocrítico y de construcción colectiva.... La justiciabilidad para que adquieran también rango formal y puedan ser seguidos con transparencia, juzgados, resueltos. De las experiencias conocidas en la región, este tipo de estrategias están muy poco desarrolladas en el campo de la educación. Posiblemente, uno de los mejores impulsores y actores son los representados por Campaña Latinoamericana por el Derecho a la Educación (CLADE). ${ }^{11}$

Dos espacios se presentan como especialmente fértiles para estos avances. Por una parte, el territorio macro entre las grandes representaciones pero que adquieren visos de compromisos públicos 
Articulaciones público-privada para la oferta educativa...

nítidos, simbólicos y seguros de ser seguidos desde varios ángulos. Y, por otra, el territorio local, en donde las cercanías de los actores se vuelven propicias para una participación mayor.

La propuesta viene cargada de un sello de tensiones, como la vida misma. Tal vez es hora de reconocerlas, acogerlas y trabajar con ellas, sin ingenuidades ni prejuicios paralizantes. Un buen momento para recuperar en la práctica y en el lenguaje el sentido de lo político. Mientras la política siga siendo solo delegada en altas esferas, corre mucho riesgo. Es hora de atraerla para el mundo cotidiano, para todos los actores, para los pequeños pasos y las grandes conquistas. Hacia la politización de la vida.

Para terminar, parece indispensable considerar este tema a la luz del fenómeno de la crisis económica que viven nuestras sociedades latinoamericanas. Una crisis de carácter estructural que no deja área sin ser afectada y cuya recuperación será larga y compleja. El riesgo de retroceder en logros educativos que han costado mucho sacrificio está presente con mucha fuerza. Los costos más altos penden una vez más sobre los sectores más excluidos.

La crisis está marcando ya el desarrollo del área educativa, y en dos sentidos complementarios. Uno directo por la disminución de las inversiones en el sector (aunque se mantengan los porcentajes del PIB) y otro indirecto por sus efectos en la nutrición, la salud, el empleo infantil, etc. Las alianzas público-privadas en educación no serán inmunes a los impactos negativos.

El primer escenario, visible ya en algunos lugares, es la disminución de la inversión del sector privado empresarial en educación, lo que revela la volatilidad y limitaciones de sustentabilidad que ha tenido. En momentos de crisis, los gastos no esenciales ni estratégicos para el bien o servicio tienden a contraerse y las metas de sobrevivencia y corto plazo a privilegiarse. Las empresas actúan con más precauciones o marcan un compás de espera.

Las inversiones en educación, por su naturaleza menos visible y sus largos plazos de impacto, son muy vulnerables en estas situaciones. Las inversiones en espectáculos, en salud, en áreas estratégicas de la salud o de la cultura, se tornan más atractivas si se trata de posicionar o reformar marca junto con la disminución de gastos (también existen exenciones de impuestos para el área cultural). El 
trabajo del Estado para sostener las colaboraciones se torna muy complejo, más aun cuando no ha existido experiencia previa en propuestas sostenibles.

El segundo escenario, bastante difícil, es el de convertir la situación en oportunidad. Sensibilizar sobre la necesidad de atacar las situaciones estructurales e implementar políticas de largo plazo que involucren a toda la sociedad. Persuadir que el desarrollo sustentable tiene como pilar central a la educación, pero que ella no se transforma sino con procesos y recursos sistemáticos de largo aliento. Demostrar que la inversión en educación no solo es un imperativo ético sino también un "buen negocio", como lo muestran múltiples estudios. ${ }^{12}$

Ojalá estas nuevas corrientes nos encuentren preparados y a la altura. Las alianzas público privadas pueden trascender lo local y lo nacional, para asumir dimensiones subregionales y regionales. El “sálvese quien pueda" sigue siendo una amenaza, tan fuerte como la esperanza de afrontar los nuevos signos de la vida en alianza, desde las alianzas... va más allá del simplista ganar-ganar o suma de fuerzas... incorpora todo un nuevo sentido y posición frente a la vida social... ver y pensar la vida desde las articulaciones políticas, no solo en un momento de negociación sino en todo el proceso... ver la acción social con los otros desde la solidaridad.

Recebido em julho de 2009 e aprovado em agosto de 2009.

\section{Notas}

1. El sentido y alcance de las articulaciones se desarrollan en el siguiente acápite del texto.

2. El fomento de alianzas ha sido planteado con vigor en acuerdos mundiales impulsados por UNESCO, OEA, OEI, eventos regionales como PRELAC; instancias subregionales y nacionales.

3. Algunos foros fueron impulsados por los estados como estrategia de apoyo a EPT, en general con poco éxito. La mayoría - que suscriben EPT y mucho más - se han generado desde la sociedad civil y con carácter autónomo. Son muy activos en Perú, Brasil, Argentina, Colombia, Ecuador. Asumen diversos nombres.

4. El poder de estos Ministerios es variable. Hacienda establece finalmente los techos de recursos con poca flexibilidad. Educación suele acudir a otras fuentes para emprender innovaciones.

5. Varias empresas españolas lideran el campo de la RSE en general y en educación también. Los aportes de las telefónicas y bancarias en becas y primera infancia son significativos y se extienden a varios países. Experiencias emblemáticas de RSE pueden consultarse en Forum Empresa o Acción RSE.

Educ. Soc., Campinas, vol. 30, n. 108, p. 699-715, out. 2009 
Articulaciones público-privada para la oferta educativa...

6. Son significativas y de avanzada las propuestas que al respecto hace el Proyecto Regional de Educación para América Latina y el Caribe (PRELAC), tanto en sus declaraciones del 2002 en Cuba, como 2007 en Argentina.

7. RSE, visión de los negocios que incorpora el respecto por los valores éticos, las personas, las comunidades y el medio ambiente (Fondo de Educación del BSR y del Forum Empresa).

8. Forum Empresa aglutina a 19 conglomerados nacionales de RSE en la región. Sus experiencias y propuestas muestran la variedad pero también una evolución sostenida hacia alianzas estratégicas.

9. El llamado Partnership for education es una expresión de las alianzas a nivel internacional entre sectores de la empresa privada e instituciones internacionales ligadas a la educación.

10. Ver Educación de calidad para todos: un asunto de derechos humanos, propuesta de PRELAC II (UNESCO, 2007).

11. CLADE se integra al momento con colectivos de 18 países y 6 redes subregionales.

12. Los estudios ligados al costo del analfabetismo, auspiciados por UNESCO en varios países de centroamérica y sudamérica, prueban fechacientemente los efectos económicos positivos que tiene la inversión en alfabetización para los individuos, familias y sociedad.

\section{Referencias}

ACOSTA, A. et al. Hacia un paco social fiscal por la educación, la salud y la inclusión social: save the children UK y CARE. Quito, 2008.

BRAVO, N. Acerca de los nuevos paradigmas en educación. Bogotá: Convenio Andrés Bello, 1997.

CAMPAÑA LATINOAMERICANA POR LA DEFENSA DEL DERECHO A LA EDUCACIÓN (CLADE). Posicionamento politico. São Paulo, 2008-2009.

CHILE. Acción empresarial. El ABC de la responsabilidad social empresarial en Chile y en el mundo. Santiago del Chile, 2009.

COMISIÓN ECONOMICA PARA AMERICA LATINA Y EL CARIBE (CEPAL). Invertir mejor para invertir más. Santiago del Chile: CEPAL/UNESCO, 2005.

COMISIÓN ECONOMICA PARA AMERICA LATINA Y EL CARIBE (CEPAL). Cohesión social, inclusión y sentido de pertenencia en América Latina y el Caribe. Santiago del Chile: CEPAL/UnESCO, 2007. CONSEJO DE EDUCACIÓN DE ADULTOS DE AMERICA LATINA (CEAAL). La Piragua: Revista Latinoamericana de Educación y Política. México, DF. 
ECUADOR. Contrato Social por la Educación. Posicionamiento y declaraciones politicas. Quito, 2006-2009.

EDUCACIÓN COMPROMISO DE TODOS. Herramientas para una política pública de educación. Bogotá, 2006.

FONDO DE EDUCACIÓN DEL BSR y FORO EMPRESA. ¿Qué es la RSE? Santiago del Chile, 2009.

FORO EDUCATIVO DEL PERÚ. Declaraciones y propuestas, 20072009.

FORO DE EDUCACIÓN DE CALIDAD PARA TODOS DE CHILE. Declaraciones y estudios, 2008.

FORO LATINOAMERICANO DE POLÍTICAS EDUCATICAS (FLAPE). Chile, 2008-2009.

MARTÍN CARRETERO, J. Participación de la sociedad civil y cohesión social. [S.l.]: FIAAP; Eurosocial, 2008.

NEIROTTI, N. De la experiencia escolar a las políticas públicas: proyectos locales de equidad educativa en 4 países de América Latina. Buenos Aires: IIPE-UneSCO, 2008.

PALMA, A. Las politicas públicas que no contribuyen a la cohesión social. [S.1]: FIAAP; Eurosocial, 2008.

PROYECTO REGIONAL DE EDUCACIÓN PARA AMÉRICA LATINA Y EL CARIBE (PRELAC). Proyecto y modelo de acompañamiento. La Habana, Cuba, 2002. Educación de calidad: un asunto de derechos humanos, Santiago, Chile, 2007.

RIVERA, J. Políticas públicas y participación. Ponencia Managua, 2007.

RIVERO, J. La articulación Estado sociedad civil en función de la mejor calidad educativa demandada en Dakar. Ponencia Managua, 2007.

TORO, B. Cooperación público privada en educación. Ponencia España, 2006.

WORLD ECONOMIC FORUM. Partnership for Education. [S.1.]: UNESCO/World Economic Forum, 2007- 2009. 